Ann. Zootech., I966, 15 (4), 343-35I.

\title{
ÉTUDE COMPARATIVE DE FARINE ET DE GRANULÉS CONCERNANT LA CONSOMMATION, LE GAIN DE POIDS ET L'EFFICACITÉ ALIMENTAIRE CHEZ DES PORCELETS SOUS LA MÈRE $\left({ }^{1}\right)$
}

\author{
R.-L. VAN SPAENDONCK et F.X. VANSCHOUBROEK
}

Laboratoire de Nutrition animale de l'Université de Gand, Belgique

\section{SOMMAIRE}

L'étude des préférences alimentaires de 85 portées de porcelets pour des aliments concentrés sous forme de farine ou de granulés a donné les résultats suivants :

I. Lorsqu'il y a distribution simultanée de farine et de granulés, les porcelets préfèrent les granulés.

2. La consommation de farine n'est pas inférieure à celle des granulés lorsque la farine et les granulés sont distribués séparément.

3. L'efficacité alimentaire des granulés paraît supérieure à celle de la farine.

\section{INTRODUC'TION}

La préférence alimentaire des porcelets pour des aliments concentrés sous forme de granulés ou sous forme de farine peut être étudiée de trois manières :

I. on peut mettre simultanément à la disposition des porcelets d'une même portée des granulés et de la farine;

2. on peut fournir exclusivement de la farine à une série de portées et exclusivement des granulés à une autre série ;

3. on peut fournir exclusivement de la farine à une moitié de la portée et exclusivement des granulés à l'autre moitié (split litter).

(1) Travail financé par l'Institut pour l'Jèncouragement de la Recherche scientifique dans l'Industrie et l'Agriculture (I. R. S. I. A.). 
Certains chercheurs ont employé la première méthode, d'autres se sont servis de la seconde et encore d'autres de la troisième.

La première méthode ne permet pas d'étudier les deux formes séparément quant à l'efficacité alimentaire. Dans la seconde méthode, cette possibilité existe mais elle exige un nombre de portées plus élevé, à cause de la grande variabilité de la consommation entre les portées. La troisième méthode permet aussi l'étude de l'efficacité alimentaire mais elle exige beaucoup de travail.

Il n'est pas certain que les trois méthodes conduisent aux mêmes conclusions. Il n'est pas certain en effet qu'une consommation alimentaire plus élevée trouvée en utilisant la première méthode se répète également avec la seconde et la troisième méthode.

Ce sont principalement des chercheurs français qui ont expérimenté la méthode de présentation simultanée de granulés et de farine aux mêmes portées.

En mettant simultanément à la disposition de 25 portées de race Large White des granulés et de la farine, SAIMON-LEI.AGNEUR et coll. (I955) ont trouvé que la consommation de granulés était en moyenne de 6,4 fois supérieure à celle de farine. Les fluctuations étaient très élevées suivant la composition des aliments. Sur 4 groupes comparés ils ont trouvé un groupe dont la consommation de granulés était 3,I fois supérieure à la consommation de farine, et un autre groupe dont la consommation était I3, I fois supérieure.

Dans les expériences qui vont suivre des chercheurs allemands, anglais et américains ont donné soit des granulés, soit de la farine.

BRAUDE et coll. (I960) en fournissant exclusivement de la farine à une série de portées et exclusivement des granulés à une autre série, trouvèrent jusqu'à l'âge de 8 semaines une consommation moyenne de $8,66 \mathrm{~kg}$ de granulés et $7,4^{8} \mathrm{~kg}$ de farine de telle sorte que la consommation de granulés était de I 8 p. Ioo supérieure à celle de farine. Les gains de poids s'élevaient respectivement à I 4,6 et I $4,2 \mathrm{~kg}$, de sorte que l'efficacité alimentaire, sans tenir compte de la consommation de lait, était respectivement de 0,59 et 0,53 .

TEAGUE et WiLsor (I957) ayant fait une expérience analogue avec 46 portées n'ont trouvé aucune différence significative entre la farine et les granulés.

WrTT et coll. (I957) en fournissant exclusivement de la farine à une moitié de la portée et exclusivement des granulés à l'autre moitié (split litter), ont réparti les porcelets par portée en deux moitiés comparables. I e premier groupe comprenant 32 porcelets n'a reçu que des granulés. La consommation par tête des porcelets du groupe granulés était plus faible que celle du groupe "farine " $(8,89$ contre $9,85 \mathrm{~kg})$, ils avaient une croissance supérieure ( 10,2 contre $9,8 \mathrm{~kg}$ ) bien que consommant moins d'aliment par $\mathrm{kg}$ de poids $(0,87$ contre I, oI $\mathrm{kg})$.

Cet aperçu de la littérature fait ressortir que si l'on soumet les porcelets à un choix entre farine et granulés, leur préférence va atıx granulés, mais qu'il y a peu de différence dans la consommation de farine ou de granulés si l'on supprime ce choix.

La validité de cette hypothèse a été testée dans l'expérience suivante, en même temps que l'effet du pressage sur l'indice de consommation. 


\section{MATÉRIEL, ET TECHNIQUES}

Dans une première série les porcelets de chaque portée disposaient simultanément de farine sèche et de granulés.

Dans une seconde série un groupe de portées disposait uniquement de farine et l'autre groupe exclusivement de granulés.

Pour les deux séries la composition de la ration était identique (tabl. I).

TABLEAU I

Composition de l'aliment concentré pour porcelets

\begin{tabular}{|c|c|}
\hline Matières premières & $\mathrm{kg}$ \\
\hline Lait écrémé en poudre $\ldots \ldots \ldots \ldots \ldots \ldots \ldots \ldots$ & $\tilde{5}$ \\
\hline Avoine décortiquée, étuvée $\ldots \ldots \ldots \ldots \ldots \ldots \ldots$ & 20 \\
\hline Orge décortiquée, non étuvée $\ldots \ldots \ldots \ldots \ldots \ldots \ldots$ & 20 \\
\hline Farine de viande, $65 \%$ mat. azot. ........... & 3,5 \\
\hline Farine de hareng, $70 \%$ mat. azot........... & 7 \\
\hline Glutenglucose de maïs $(60 \%$ gluten $+40 \%$ glucose $)$ & 10 \\
\hline Farine de tourteau de soja, solvant, grillé .......... & 15 \\
\hline 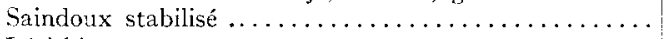 & 5 \\
\hline Lécithine $\ldots \ldots \ldots \ldots \ldots \ldots \ldots \ldots \ldots \ldots \ldots \ldots \ldots \ldots$ & 1 \\
\hline Sucre de betterave, dénaturé (1) .............. & 8 \\
\hline Prémélange minéral $\left(^{\mathbf{2}}\right) \ldots \ldots \ldots \ldots \ldots \ldots \ldots \ldots \ldots \ldots \ldots$ & 0,5 \\
\hline Prémélange de vitamines et d'antibiotiques $\left({ }^{3}\right) \ldots \ldots$. & 5 \\
\hline
\end{tabular}

(1) Le sucre de betterave dénaturé est composé de 86 p. 100 de sucre de betterave, 11 p. 100 de farine de luzerne et 3 p. 100 de $\mathrm{NaCl}$.

(2) Le prémélange minéral est composé de : $\mathrm{Na}_{2} \mathrm{SeO}_{3} 5 \mathrm{H}_{2} \mathrm{O}: 0,0035 \%$; $\mathrm{CoSO}_{4} 7 \mathrm{H}_{2} \mathrm{O}: 0,2 \% ; \mathrm{ZnSO}_{4} 7 \mathrm{H}_{2} \mathrm{O}: 4 \% ; \mathrm{CuSO}_{4} 5 \mathrm{H}_{2} \mathrm{O}: 10 \% ; \mathrm{FeSO}_{4} 7 \mathrm{H}_{2} \mathrm{O}$ : $6 \% ; \mathrm{MnSO}_{4} 5 \mathrm{H}_{2} \mathrm{O}: 3 \%$; NaCl iodé : $30 \% ; \mathrm{Ca}_{2} \mathrm{H}_{2}\left(\mathrm{PO}_{4}\right)_{2}: 46,8 \%$.

(3) Le prémélange de vitamines et d'antibiotiques est composé de : vitamine A : $600000 \mathrm{UI}$; vitamine $\mathrm{D}_{3}: 200000 \mathrm{UI}$; riboflavine : $500 \mathrm{mg}$; panthothénate de $\mathrm{Ca}: 100 \mathrm{mg}$; acide nicotinique : $4000 \mathrm{mg} ; \alpha$-tocophérol : $2000 \mathrm{mg}$; acide ascorbique : $10000 \mathrm{mg}$; butyl hydroxytoluène : $10 \mathrm{~g}$; terramycine : $5 \mathrm{~g}$; poudre de lait écrémé q.s.p. $5 \mathrm{~kg}$.

Les granulés sont fabriqués au moyen d'une presse à froid. La température de la matrice était de $5^{\circ} \mathrm{C}$, environ. Les granulés sont modérément consistants. Le diamètre est de $5 \mathrm{~mm}$, la longueur de ${ }_{5} 5 \mathrm{~mm}$. La teneur en eau est la même pour la farine et les granulés, c'est-à-dire i I p. Ioo.

Les porcelets appartiennent tous à la race belge. Après la naissance ils séjournent une dizaine de jours dans la maternité. Ensuite la truie et les porcelets sont dirigés vers une porcherie d'élevage, faisant partie du même groupe de porcheries que la maternité. Chaque semaine les porcelets sont pesés. Ils sont sevrés à l'âge de 8 semaines. A partir de la $3^{\mathrm{e}}$ semaine les porcelets disposent à discrétion du concentré dans des mangeoires en bois.

La truie n'a pas accès aux aliments des porcelets et pendant la distribution d'aliments à la truie les porcelets n'ont pas accès aux aliments de la truie. Le troisième jour après la naissance les porcelets de la seconde série d'essais reçoivent une injection de $200 \mathrm{mg}$ de fer sous forme de dextrane.

Chaque semaine et jusqu'au sevrage la consommation de farine et de granulés est pesée séparément et exprimée par porcelet. Les porcelets disposent d'abreuvoirs automatiques. 
Pour la seconde série d'essais la production laitière des truies a été déterminée, suivant une méthode décrite antérieurement (VAN SPAENDONCK et VANSCHOUBROEK, I964a). La composition du lait a également été déterminée, ceci ayant pour but d'étudier l'efficacité alimentaire pour les deux formes de concentré.

Nous avons calculé (VAN Spaendonck et Vanschoubroek, $1964 b$ ) que pour un gain de poids d'un kilo il faut fournir 4 I $30 \mathrm{kcal}$ brutes sous forme de lait. En divisant la quantité de calories de lait produite par porcelet par 4 I 30 on peut calculer le nombre de $\mathrm{kg}$ de porcelets, supposé produit par le lait. En soutrayant ce nombre de kilos de porcelets du gain de poids total, on obtient le nombre de $\mathrm{kg}$ de poids de porcelets élaboré par le concentré. Ceci permet d'estimer l'efficacité de l'utilisation de la farine ou des granulés.

Pour la première série d'essais 39 portées ont été utilisées avec en moyenne 9,7 porcelets à la mise bas. A partir de la $3^{\mathrm{e}}$ semaine les porcelets disposent simultanément de farine et de granulés.

Pour la deuxième série d'essais 46 portées ont été utilisées; dès le début de la troisième semaine 24 portées reçoivent uniquement de la farine, 22 autres exclusivement des granulés.

Pour les calculs statistiques et autres nous avons toujours utilisé les moyennes par porcelet des différentes portées. Le test de signification de la différence de deux moyennes a été effectué à l'aide du test de Student.

\section{RÉSULTATS}

r. Comparaison de la consommation de farine et de granulés lorsque les deux formes d'aliments sont mises simultanément à la disposition des porcelets

Le nombre moyen de porcelets par semaine et par portée, ainsi que la consommation alimentaire hebdomadaire moyenne par porcelet, pour les différentes portées figurent au tableau 2 .

TABLEAU 2

Consommation moyenne hebdomadaire de farine et de granulés par porcelet pour les portées de la première série d'essais

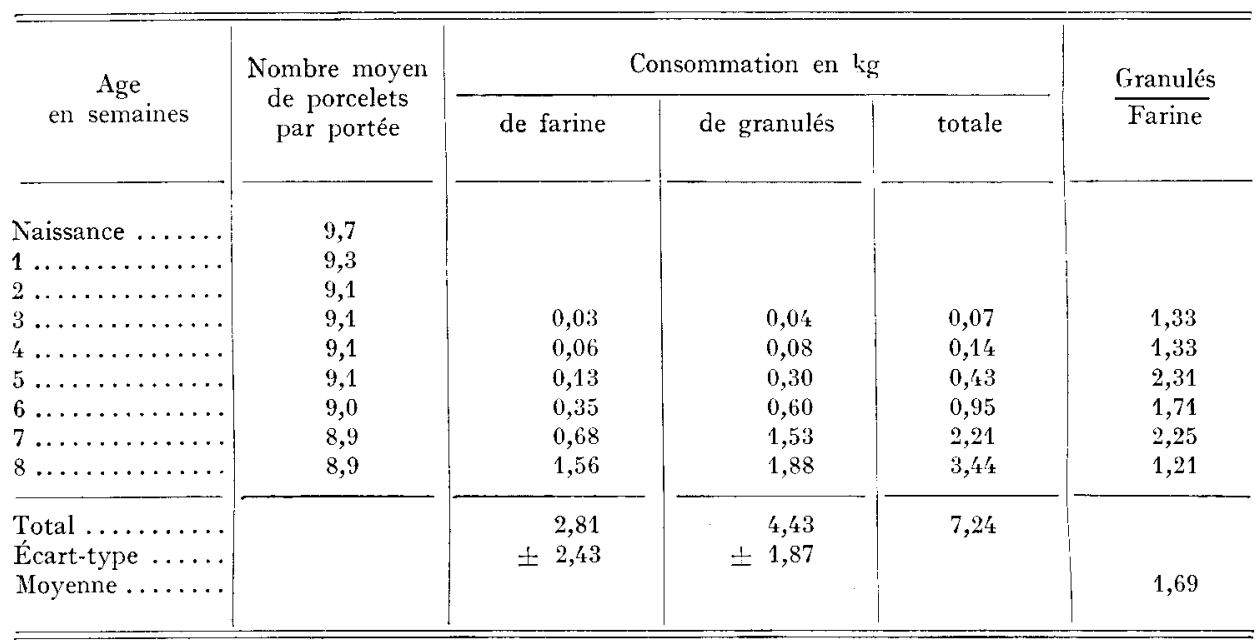

Comme 1'indique ce tableau, sur une moyenne de 9,7 porcelets par portée à la mise bas il en reste 8,9 au moment du sevrage à 8 semaines. 
A partir de la $3^{\text {e }}$ semaine jusqu'au sevrage la consommation moyenne est de 2,8I $\mathrm{kg}$ de farine et de 4,43 $\mathrm{kg}$ de granulés, soit au total 7,24 $\mathrm{kg}$ d'aliments concentrés. Ainsi la consommation de granulés est $\mathrm{I}, 7$ fois supérieure à celle de la farine. La différence dans la consommation de farine et de granulés est très significative $(\mathrm{P}<\mathrm{O}, \mathrm{OI})$. Le tableau 2 nous montre également que chaque semaine la consommation de granulés est supérieure à celle de farine mais que le rapport entre la consommation hebdomadaire de granulés et de farine varie d'une semaine à l'autre.

La corrélation entre la consommation de farine et de granulés est de $0,4 \mathrm{I}$, ce qui signifie que les porcelets qui consomment le plus de granulés consomment également le plus de farine, mais pas toujours dans la même proportion.

La variation dans la consommation de farine s'élève à $86 \mathrm{p}$. roo de la moyenne, celle de la consommation de granulés à $42 \mathrm{p}$. Ioo de la moyenne. La variation dans la consommation de la farine est donc 2 fois plus grande que celle de la consommation de granulés. Cela revient à dire que si les porcelets peuvent choisir, certaines portées consomment beaucoup de farine, tandis que d'autres en consomment peu.

Le gain de poids moyen de la naissance jusqu'au sevrage s'élève à $14,49 \pm$ $3,36 \mathrm{~kg}$.

2. Comparaison de la consommation de farine et de granulés mis à la disposition des porcelets, soit exclusivement sous forme de farine soit exclusivement sous forme de granulés

Le nombre moyen de porcelets par semaine et par portée, ainsi que la consommation moyenne hebdomadaire de farine et de granulés par porcelet et pour les deux groupes de portées figurent au tableau 3 .

TABLEAU 3

Consommation hebdomadaire moyenne de farine et de granulés par porcelet pour les portées de la deuxième série d'essais

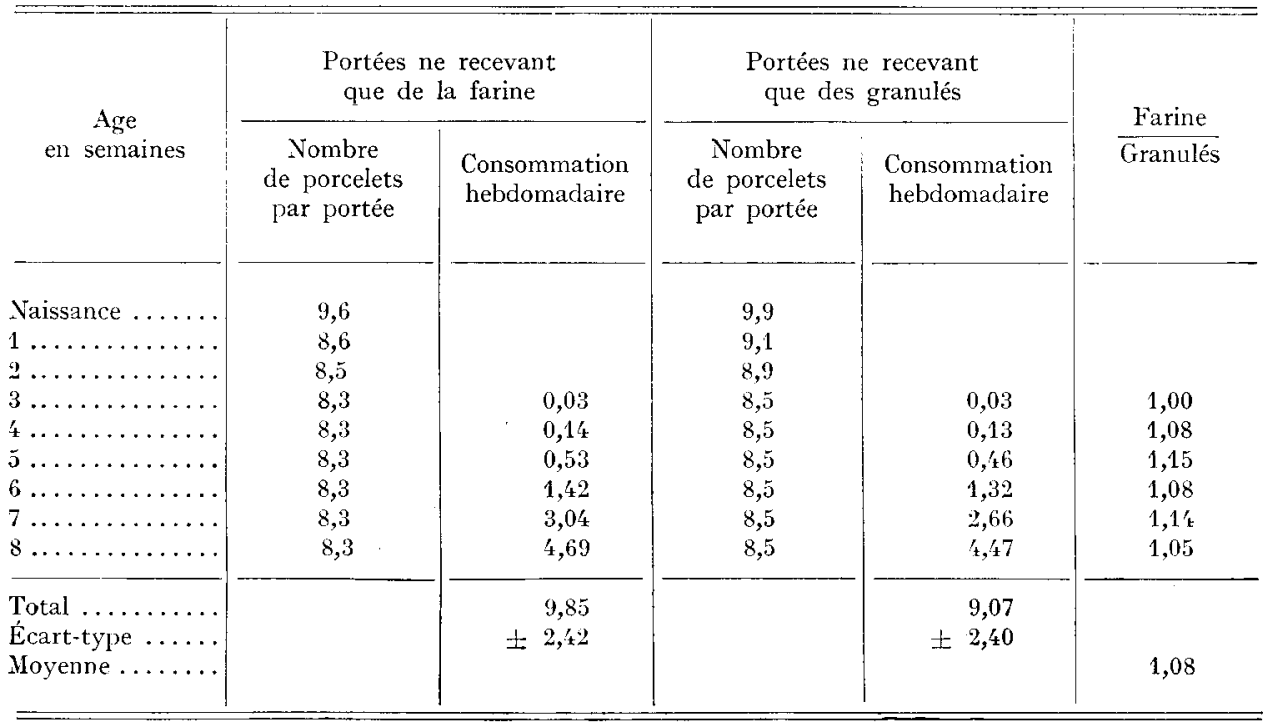


La consommation alimentaire moyenne de la $3^{\mathrm{e}}$ à la $8 \mathrm{e}$ semaine s'élève à $9,85 \mathrm{~kg}$ pour les porcelets ne recevant que de la farine et à $9,07 \mathrm{~kg}$ pour les porcelets ne recevant que des granulés. Le rapport moyen entre la consommation de farine et de granulés s'élève à $\mathrm{I}, 08$. Ce rapport change peu au cours de la période d'allaitement.

Le fait que les consommations de farine et de granulés soient identiques pour la $3^{\mathrm{e}}$ semaine de la vie nous permet de conclure que le moment où les porcelets commencent à prendre de la nourriture consistante n'est pas influencé par la forme de l'aliment.

Les résultats au sujet du gain de poids et de l'efficacité alimentaire figurent au tableau 4 .

\section{TABLEAU 4}

Consommation moyenne totale et gain de poids total par porcelet pour les différenles portées

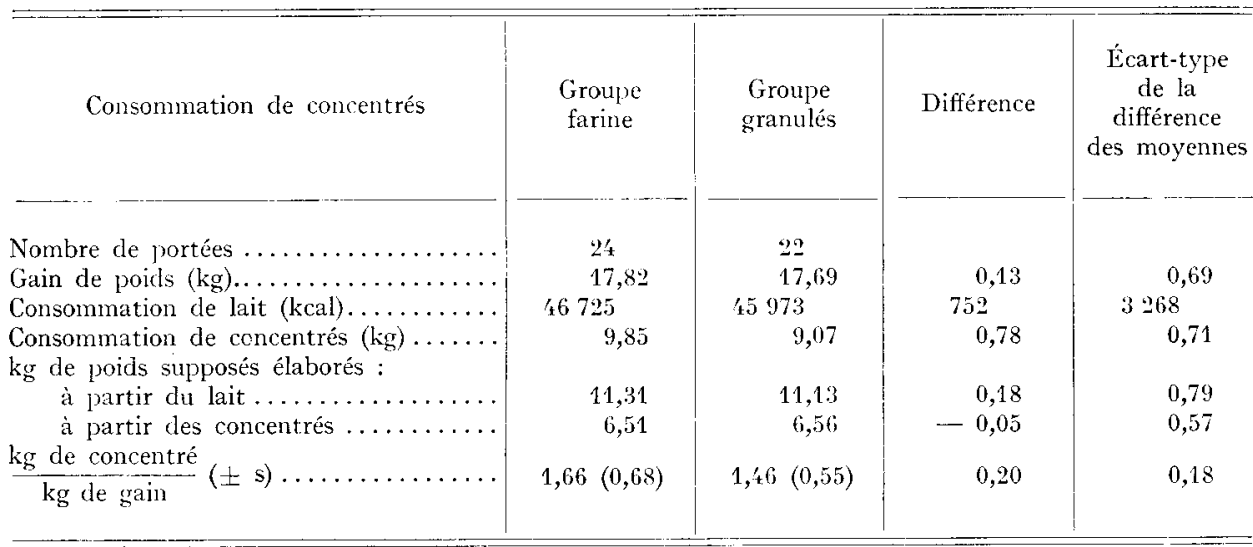

Le gain de poids de la naissance jusqu'au sevrage est $\mathrm{I}_{7}, 82 \mathrm{~kg}$ pour les porcelets ne recevant que de la farine et $\mathrm{I} 7,69 \mathrm{~kg}$ pour les porcelets ne recevant que des granulés. Il existe une différence dans la consommation de concentrés sous forme de granulés ou de farine. Cette différence, qui est de 7,9 p. Ioo, n'est pourtant pas significative $(\mathrm{P}=0,3)$.

Entre les deux groupes il n'y a pratiquement pas de différence en ce qui concerne la consommation de lait.

Le nombre total de $\mathrm{kcal}$ consommées par porcelet s'élevait à 46725 pour le groupe "farine " et à 45973 pour le groupe " granulés ". D'après notre calcul (voir plus haut) le nombre de $\mathrm{kg}$ de poids de porcelet supposé élaboré par le lait s'élève à II,3I pour les porcelets du groupe "farine " et à II,I3 pour les porcelets du groupe " granulés ". Le nombre de kilos supposé élaboré par l'aliment concentré serait alors resp. 6,5 I et 6,56 . Aucune de ces différences n'est significative. Les porcelets du groupe " farine » utilisent $\mathrm{I}, 66 \mathrm{~kg}$ de farine pour un $\mathrm{kg}$ de gain, les porcelets du groupe " granulés » I,46 kg de granulés. Cette différence n'est cependant pas significative $(\mathrm{P}=0,3)$. La variation de l'indice de consommation est très grande ce qui est démontré par les écarts-types 0,68 et 0,55 . 


\section{DISCUSSION}

Les résultats concernant la consommation alimentaire correspondent bien aux résultats mentionnés dans la littérature. Nous avons constaté comme SALMONLEGAGNEUR et coll. (I955) et NELSON et coll. (I953) que les porcelets préfèrent les granulés à la farine, s'ils disposent d'un aliment sous les deux formes.

Les résultats des différents chercheurs ne correspondent pas toujours dans le cas où la nourriture a été fournie soit sous l'une soit sous l'autre forme.

BRAUDE et coll. ( $\mathrm{r} 960$ ) constataient une consommation plus élevée de granulés que de farine. Mais dans leur expérience $5 \mathrm{p}$. Ioo de céréales de la farine étaient remplacés par 5 p. Ioo de melasse dans l'aliment granulé et ainsi les deux formes n'avaient pas la même composition. TEAGUE et WILSON (I957) n'ont pas trouvé de différence. Dans les essais de WITT et coll. (I957) la consommation de farine était plus élevée que celle de granulés. Cej auteurs ont constaté que le moment où les porcelets commencent à prendre de la nourriture consistante n'est pas influencé: par la forme de l'aliment. Les résultats de WITT et coll. sont donc en concordance avec les nôtres. Comme dans la présente expérience les deux formes d'aliment contenaient du sucre (IO p. IOO).

Les différences trouvées par les auteurs cités comme par nous-mêmes lors d'une distribution séparée ne sont en aucun cas significatives.

Quoique obtenues chez des porcelets sevrés précocement (à 2 semaines) les études de JENSEN et coll (I959) et de NELSON et coll. (I953) sont intéressantes. I,es premiers trouvaient une consommation journalière de $23 \mathrm{I} g$ de "granulés " et de $240 \mathrm{~g}$ de "farine ", soit une différence négligeable. Le groupe " granulés " accusait cependant une efficacité alimentaire meilleure $(I, 5 \mathrm{I})$ que ceux du groupe "farine " $(\mathrm{I}, 75)$. Les derniers trouvaient une consommation journalière de $472 \mathrm{~g}$ de granulés et de $38 \mathrm{I} g$ de farine. Cela signifie que la consommation de granulés était de $25 \mathrm{p}$. Ioo supérieure à celle de farine. La consommation de granulés était cependant fonction du taux de sucre. Avec 7,7 p. Ioo et I5 p. Ioo de sucre, la consommation de granulés était respectivement $60 \mathrm{p}$. Ioo et $33 \mathrm{p}$. Ioo plus élevée que celle de farine. Avec 7,5 p. Ioo de mélasse les résultats étaient intermédiaires. Pour les portées ne recevant pas de sucre, les porcelets consommaient ro p. roo de farine de plus que de granulés.

\section{CONCLUSIONS}

On peut conclure des données citées ci-dessus que les porcelets préfèrent les granulés lorsque la distribution est simultanée; mais la consommation de farine n'est pas inférieure à celle des granulés lorsque la farine ou les grantulés sont distribués séparément.

La consommation relative de granulés et de farine semble bien déterminée par les propriétés chimiques et physiques des granulés. Par propriétés chimiques 
on comprend la composition de l'aliment et les modifications dues aux influences mécaniques et thermiques dans la presse; par propriétés physiques on entend la forme (diamètre et longueur) et la consistance des granulés. A ce sujet les résultats de NELSON et coll. (r953) concernant l'influence de l'addition de sucre sont rappelés.

Aumaitre et Salmon-Legagneur (I96I) ont étudié les préférences des porcelets pour des granulés de 3 dimensions, dont les diamètres étaient respectivement de 7,5 et $2,5 \mathrm{~mm}$ et les longueurs de 20 , Io et $5 \mathrm{~mm}$ et ont trouvés que les porcelets montrent une préférence très nette pour les granulés à faible diamètre.

L'efficacité alimentaire meilleure dans le cas de granulés, quoique non significative, nous semble logique, entre autre pour les raisons suivantes : a) Moins de perte pendant la distribution et pendant les repas; $b$ ) Meilleure digestion causée par le hachage des fibres cellulosiques et permettant un accès plus facile des enzymes digestifs au contenu des cellules (SPERLing, I955; DAmmers, I957, 1964) ; dégradation partielle des grains d'amidon sous l'influence de la chaleur permettant une meilleure action de l'amylase (JENSEN et coll., I959).

Il n'est pas encore suffisamment établi que l'amélioration éventuelle de l'indice de consommation compense l'augmentation du prix de revient due au pressage.

Il est même possible, que dans certains cas le pressage ait une influence défavorable sur l'efficacité alimentaire. Le granulé peut être trop dur, entraînant un travail supplémentaire de mastication et une augmentation de la fraction non digérée (BRAUDE et coll., I960). Le grranulé peut être également pressé à des températures trop élevées pouvant entraîner la dénaturation des protéines et des vitamines.

Ręu pour publication en septembre 1966.

\section{SUMMARY}

COMPARISON OF MEAL AND PELLETS IN RELATION TO FEED CONSUMPTION, WEIGHT GAIN AND FEED EFFICIENCY OF SUCKLING PIGS

Although the results of different experiments are not always in agreement, the data of the literature suggest that piglets which have a choice between meal and pellets, prefer pellets, but that the physical form of the feed has only little influence on the feed consumption of piglets, having the disposal of only meal or pellets each separately.

To check the validity of this hypothesis the present experiment has been carried out.

In a first series of experiments on 39 litters, the piglets of the same litter had access to both meal and pellets.

In a second series 24 litters received only meal, whereas 22 other litters received only pellets.

In booth series the piglets, all belonging to the Belgian I.andrace, were weighted with weekly interval and weaned at 8 weeks of age.

In order to compare the efficiency of feed utilization of both forms, the milk yield and milk composition was determined in the second series.

For the first series, the average consumption amounts to $7.24 \mathrm{~kg}$ of creep feed namely $2.8 \mathrm{I} \mathrm{kg}$ as meal and $4.43 \mathrm{~kg}$ as pellets, or $\mathrm{I} .7$ times as much pellets as meal. The correlation between the consumption of meal and the consumption of pellets was $0.4 \mathrm{I}$. This means that litters, which consume more pellets are also consuming more meal, but not to the same degree. The variation of the meal consumption was 2.2 times higher than that of pellets.

In the second series, when the creep feed was given either as meal or as pellets, meal consumption per piglet was higher than that of pellets $(9.85 \mathrm{vs} 9.07 \mathrm{~kg})(\mathrm{P}=0.3)$. The difference in weight gain of piglets from birth to eight weeks was very small for " meal " and "pellets " piglets (I 7.82 vs I $7,69 \mathrm{~kg})$. 
Moreover, the "meal » piglets consumed on the average $46725 \mathrm{kcal}$, whereas the "pellets" piglets 45973 kcal during the suckling period.

In a previous study it was found that $4130 \mathrm{kcal}$ as milk was required for one kilogram of weight gain of piglets. When this was assumed it was calculated that $6.5 \mathrm{I} \mathrm{kg}$ was formed from the creep feed for " meal " piglets and $6.56 \mathrm{~kg}$ for " pellets " piglets. The efficiency of feed utilisation then would be 1.66 for the "meal "piglets and 1.46 for the "pellets " piglets, the standard deviation of mean being 0.68 resp. 0.55 .

None of the differences between weight gain, energy consumption, milk conversion efficiency, creep conversion efficiency were statistically significant.

From the experiment can be derived that, although piglets prefer pellets upon meal, pelleting the creep feed does not increase total feed consumption. There are some indications however that feed conversion efficiency can be improved by pelleting.

\section{RÉFÉRENCES BIBLIOGRAPHIQUES}

Aumaitre A., Salmon-Legagnedr E., ig6r. Les préférences alimentaires du porcelet. V. Comparaison de divers modes de distribution de l'aliment. Arn. Zoolech., 10, 197-203.

Braude R., Townsend M. J., Rowell J. G., ig6o. A comparison of meal and pelleted forms of creep feed for suckling pigs. J. agric. Sci., 54, $274^{-277}$.

Dammers J., 1957. Verteringsproeven met varkens, vergelijking van varkenskorrels met varkensmeel. Landbowovoorlichting, 14, $293^{-295}$.

Dammers J., 1964. Verteringsstudies bij het varken. Faktoren van invloed op de vertering der voedercomponenten en de verteerbaarheid der aminozuren. Proefschrift Univ. Leuven, Landbouwinstituut.

Jensen A. H., Becker D. E., Norton H. W., Terrill, S. W., i959. The effect of pelleting upon nutritive value of rations for pigs weaned at two weeks of age. J. anim. Sci., 18, I $49 \mathrm{r}$ (abstr.).

Nelson L. F., Catron D. V., 1953. Baby pigs have a sweet tooth. Feedsiuffs, 25, I6-i8.

Salmon-Legagneur E., FÉvrier R., 1955. Las préférences alimentaires du porcelet. I. Influence du mode de présentation des aliments granulés ou farine. Ann. Zootech., 4, 215-218.

Sperding L., 1955. Pressfutter in der Aufzucht von Ferkeln. Fuller u. Futlerung, 54, 433-435.

Teague H. S., Wilson R. F., 1957. Meal versus the pelleted form of creep feed. Creep feeds for suckling pigs, the effect of oast Hull Fiber, Sugar and pelleting. Res. Circ, 46, Ohio Agric. Sta.

Van Spaendonck R. L., Vanschoubroek F. X., I964a. Dztermination of the milk yield of sows and correction for loss of weight due to metabolic processes of piglets during suckling. Animal Prod., 6, I I9-I 23.

Van Spaendonck R. L., Vansciroubroek F. X., $1964 b$. L'efficience alimentaire chez les porcelets pendant la périede d'allaitement. Ann. Zootech., 13, $n^{\mathbf{0}}$ hors-série 1, g6-ıo6.

WITT M., ANDREAE U., SCHRöDER J., I957. Einfluss von zuckerhaltigen und zuckerfreien Fertigfutter in Mehl und Pelletsform auf den Beginn und die Höhe der Futteraufnahme bei Ferkeln. Zuchlungskunde, 29, I $4^{2-1} 53$. 